\title{
Naringenin ameliorates LPS-induced acute lung injury through its anti-oxidative and anti-inflammatory activity and by inhibition of the PI3K/AKT pathway
}

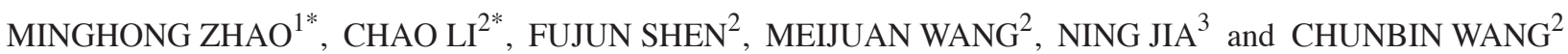 \\ ${ }^{1}$ Department of Oncology, Jianhu People's Hospital, Yancheng, Jiangsu 224700; \\ ${ }^{2}$ Department of Oncology, Yancheng Third People's Hospital, Yancheng, Jiangsu 224001, P.R. China; \\ ${ }^{3}$ Research and Development Department, Solomon Brothers Medical Institute, Wilmington, DE 19803, USA
}

Received May 13, 2016; Accepted March 24, 2017

DOI: $10.3892 /$ etm.2017.4772

\begin{abstract}
The aim of the present study was to explore the effect of naringenin on lipopolysaccharide (LPS)-induced acute lung injury (ALI) in a mouse model, as well as the underlying mechanism. The animals were randomly assigned to four groups: PBS-treated healthy control (Control), LPS-induced ALI (LPS), vehicle-treated ALI (LPS + Vehicle), and naringenin-treated ALI (LPS + Nar) group. Naringenin $(100 \mathrm{mg} / \mathrm{kg})$ was administered orally for 4 consecutive days, starting 3 days prior to induction of ALI. The survival rates of mice, lung wet/dry weight ratios, lung injury score, protein levels of bronchoalveolar lavage fluid (BALF), lactate dehydrogenase (LDH) activity in the BALF, lung myeloperoxidase (MPO) activity, the number of infiltrated neutrophils and reactive oxygen species (ROS) levels $\left(\mathrm{H}_{2} \mathrm{O}_{2}\right.$ and malondialdehyde) were assessed. In addition, the serum and BALF levels of inflammatory cytokines [tumor necrosis factor- $\alpha$, interleukin (IL)-1 $\beta$, IL-6 and macrophage inflammatory protein 2] were determined, along with the total and the phosphorylated protein levels of phosphatidylinositol 3-hydroxy kinase (PI3K) and AKT in lung tissues. The results showed that naringenin pre-treatment significantly increased the survival rate, improved histopathologic changes, alleviated pulmonary edema and lung vascular leak, downregulated the levels of ROS and reduced neutrophil infiltration as well as the levels of inflammatory cytokines in the serum and BALF. Moreover, naringenin pre-treatment reduced the total and the phosphorylated protein levels of PI3K and AKT. The present
\end{abstract}

Correspondence to: Dr Chunbin Wang, Department of Oncology, Yancheng Third People's Hospital, 75 Juchang Road, Yancheng, Jiangsu 224001, P.R. China

E-mail: wangchunbin8679@126.com

*Contributed equally

Key words: naringenin, acute lung injury, anti-oxidative, anti-inflammatory, PI3K/Akt pathway study suggested that naringenin pre-treatment ameliorated LPS-induced ALI through its anti-oxidative and anti-inflammatory activity and by inhibition of the PI3K/AKT pathway in mice.

\section{Introduction}

Acute lung injury (ALI) and the more severe acute respiratory distress syndrome (ARDS) are serious and potentially life-threatening events, which are characterized by pulmonary infiltrates, hypoxemia and edema (1). Despite tremendous advances in the prevention and treatment of ALI, the incidence of ALI is high and the mortality rate remains as high as $40 \%$ (2). Furthermore, the treatment of ALI represents an enormous financial burden to society and individuals. In addition, no effective therapeutic regimen is available for patients with this syndrome. Therefore, additional research is urgently required to explore novel pathways that may be targeted for the development of novel treatment options.

Although the pathophysiology of ALI has remained to be fully elucidated, an exaggerated inflammatory response has been reported to have a significant role (1). The inflammatory response is initiated, amplified and regulated by a complex network of cytokines and other pro-inflammatory cytokines (3). Pro-inflammatory cytokines, such as tumor necrosis factor (TNF)- $\alpha$, interleukin (IL)-1 $\beta$, IL-6 and macrophage inflammatory protein 2 (MIP2) have been demonstrated to have a role in the pathogenesis of ALI (4). Exaggerated neutrophilic infiltration is responsible for the damage of alveolar-capillary barrier (5), resulting in the non-cardiogenic pulmonary edema. In addition, oxidative stress modulated by reactive oxygen species (ROS) is involved in the pathogenesis of ALI $(6,7)$. Oxidative stress promotes the recruitment and activation of residential neutrophils and macrophages, leading to inflammatory and thereby cellular injury (8). Moreover, the phosphatidylinositide-3-kinase (PI3K)/AKT signaling pathway has been reported to negatively regulate lipopolysaccharide (LPS)-induced acute inflammatory responses in vitro and in vivo $(9,10)$. Previous studies have found that inhibition of the PI3K/AKT signaling pathway exerts a protective role in ALI (11). 
Naringenin, a flavonoid contained in citrus fruit, grapefruits and tomatoes, has been reported to exert multiple biological and pharmacological effects, such as anti-oxidative, anti-proliferative, anti-inflammatory and anti-tumor activities (12-15). Previous studies have suggested that naringenin protected normal human bronchial epithelium against LPS-induced injury (16) and improved LPS-induced ALI in rats through its anti-inflammatory, anti-oxidant, anti-nitrosative and anti-apoptotic effects (17). However, whether naringenin protects against LPS-induced ALI through the PI3K/AKT signaling pathway has remained to be investigated. Therefore, the present study explored the effects of naringenin on LPS-induced ALI, as well as the underlying signaling pathways.

\section{Materials and methods}

Animals and groups. A total of 80 adult female C57BL/6 mice (8-10 weeks of age, weighing 20-25 g) were purchased from Shanghai SLRC Laboratory Animal Co., Ltd. (Shanghai, China). All the animals were maintained under barrier conditions in single and ventilated cages with free access to standard diet and tap water, and kept under a 12-h light/dark cycle with controlled temperature $\left(22-25^{\circ} \mathrm{C}\right)$ and humidity $(50 \%)$. The mice were randomly divided into four groups $(n=20$ in each group): Phosphate-buffered saline (PBS)-treated healthy control (Control), LPS-induced ALI (LPS), vehicle-treated ALI (LPS + Vehicle) and naringenin-treated ALI (LPS + Nar). All experimental procedures were performed according to the Guide for Care and Use of Laboratory Animals published by the China National Institutes of Health and were approved by the ethics committee of Yancheng Third People's Hospital (Yancheng, China).

ALI mouse model. All mice were anesthetized with xylazine $(65$ mg/kg; Sigma-Aldrich; Merck KGaA, Darmstadt, Germany) and ketamine (150 mg/kg; Sigma-Aldrich; Merck $\mathrm{KGaA}$ ) by intravenous injection. ALI was induced by intratracheal instillation of Escherichia coli LPS (055:B5; Sigma-Aldrich; Merck KGaA, Darmstadt, Germany; $25 \mu \mathrm{g}$ in $50 \mu 1$ sterile saline) as described previously (18). The control mice received intratracheal instillation of sterile PBS alone, mice in the LPS + Vehicle group received LPS (Sigma-Aldrich; Merck KGaA) and then vehicle (PBS) treatment and mice in the LPS + Nar group were first orally administered naringenin (100 mg/kg; Sigma-Aldrich; Merck KGaA) that was dissolved in $10 \%$ Cremophor (BASF, Ludwigshafen, Germany) for 4 consecutive days, starting 3 days prior to the induction of ALI and then received LPS to induce ALI. Half of the mice $(n=10$ in each group) were anesthetized by intraperitoneal injection of thiopental $(50 \mathrm{mg} / \mathrm{kg}$; Penthotal; Ospedalia AG, Hünenberg, Switzerland) at $24 \mathrm{~h}$ after the induction of ALI for further analysis and the remaining mice ( $\mathrm{n}=10$ in each group) were used to determine the survival rate. The thorax was opened and blood samples were collected. Furthermore, a bronchoalveolar lavage (BAL) procedure was performed at least 3 times using normal saline. The blood was centrifuged at $2,000 \mathrm{x} \mathrm{g}$ for $10 \mathrm{~min}$ at $4^{\circ} \mathrm{C}$ and the serum was stored for further analysis.

Survival rate. To determine whether pre-treatment with naringenin protects against LPS-induced ALI, the survival rates were recorded at $0,24,48,72,96$ and $120 \mathrm{~h}$ after induction of ALI and a survival curve was then drawn.

Determination of lung wet/dry ratio. After sacrifice, the left lungs of the mice were harvested and the wet lungs were weighted immediately after dissection. The lung tissues were dried for 3 days at $80^{\circ} \mathrm{C}$ and were then weighed again. The wet/dry ratio was calculated by dividing the wet weight by the final dried weight (19).

Collection of BAL fluid (BALF) and cell counting. BALF was collected as previously described (20). In brief, after the animals were sacrificed, the BALF was obtained by intratracheal intubation. BAL procedures were performed using $0.5 \mathrm{ml}$ sterile saline. The BALF was centrifuged $\left(4^{\circ} \mathrm{C}\right.$, $3,000 \times \mathrm{g}, 10 \mathrm{~min})$, and the cell-free supernatants were stored at $-70^{\circ} \mathrm{C}$ for the subsequent analysis of inflammatory cytokine and protein concentrations. The erythrocytes were removed by addition of $0.8 \%$ ammonium chloride solution for $1 \mathrm{~min}$. The BALF cell pellet was re-suspended to the original volume in PBS for cell counting with a hemocytometer.

Morphological assessment of lung injury. After sacrifice, the left lungs of the mice were harvested and fixed in $4 \%$ formalin (Sigma-Aldrich) overnight at $4^{\circ} \mathrm{C}$. The lung tissues were then embedded with paraffin, sectioned $(4 \mu \mathrm{m})$ and stained with hematoxylin and eosin, followed by light microscopic observation. To determine the lung injury score, the histological changes were evaluated by an experienced pathologist blinded to the study groups. The total lung injury score was calculated as the sum of the scores for each variable (edema, neutrophil infiltration, interstitial inflammation and congestion) according to a previously described method (21). The score ranged between 0 (normal) and 4 (severe), and the average values were recorded as the semi-quantitative histological index of lung injury.

Measurement of lactate dehydrogenase (LDH) activity in $B A L F$. The LDH activity in the BALF was determined by using a commercial LDH determination kit (Sigma-Aldrich; Merck KGaA). In brief, BALF supernatants $(200 \mu \mathrm{l})$ were added to Reagent A $(2.5 \mathrm{ml})$ for $1 \mathrm{~min}$, followed by the addition of Reagent B $(100 \mu \mathrm{l})$. Absorbance at $340 \mathrm{~nm}$ was read every minute for $3 \mathrm{~min}$ and $\mathrm{LDH}$ activity was determined using LDH standards.

Measurement of total protein concentration in BALF. To assess the effect of naringenin on lung permeability, BALF total protein was measured by using a Bradford assay (Bio Rad Laboratories, Inc., Hercules, CA, USA).

Measurement of lung myeloperoxidase (MPO) activity. Frozen tissue samples of the right lower lung lobe were homogenized with RIPA buffer (Sangon Biotech Co, Ltd Shanghai, China), as a protease and phosphatase inhibitor (Thermo Fisher Scientific, Inc., Waltham, MA, USA) and sonicated. The suspensions were centrifuged at $2,430 \mathrm{x}$ g for $10 \mathrm{~min}$ at $4^{\circ} \mathrm{C}$. The MPO activity in the supernatant was determined by continuously monitoring the hydrogen peroxide $\left(\mathrm{H}_{2} \mathrm{O}_{2}\right)$-dependent oxidation of dianisidine dihydrochloride (Sigma-Aldrich; Merck KGaA). In brief, 
the supernatant $(0.1 \mathrm{ml})$ was mixed with $\mathrm{PBS}$, containing sodium dihydrogen phosphate and disodium monohydrogen phosphate plus sodium hydroxide $(2.9 \mathrm{ml}, 50 \mathrm{mmol} / \mathrm{l}, \mathrm{pH} 6.0)$ supplemented with $\mathrm{H}_{2} \mathrm{O}_{2}(0.0005 \%)$ and dianisidine dihydrochloride $(0.167 \mathrm{mg} / \mathrm{ml})$. Absorbance at $460 \mathrm{~nm}$ was measured using a spectrophotometer.

ELISA. The levels of TNF- $\alpha$, IL-1 $\beta$, IL-6 and MIP2 were measured by ELISA (R\&D Systems, Minneapolis, MN, USA) according to the manufacturer's instructions. The absorbance at $450 \mathrm{~nm}$ was measured using a microplate reader (EMax Endpoint ELISA Microplate Reader; Molecular Devices, LLC., Sunnyvale, CA, USA). The experiment was repeated at least three times.

Measurement of oxidative stress. To determine the level of oxidative stress in the lungs, the activity of hydrogen peroxide $\left(\mathrm{H}_{2} \mathrm{O}_{2}\right)$ and malondialdehyde (MDA) was assessed. In brief, the lung tissues were homogenized in $0.01 \mathrm{M}$ PBS and centrifuged at $10,000 \mathrm{xg}$ for $15 \mathrm{~min}$ at $4^{\circ} \mathrm{C}$. The supernatants were collected and the activities of $\mathrm{H}_{2} \mathrm{O}_{2}$ and MDA were measured by using an $\mathrm{H}_{2} \mathrm{O}_{2}$ kit and an MDA kit (both from Abcam, Cambridge, MA, USA) following the instructions of the manufacturer.

Western blot analysis. Protein was extracted from the tissues and the protein concentration was determined by using a BCA Protein Assay kit (Pierce; Thermo Fisher Scientific, Inc.). Equal amounts of protein (20 $\mu \mathrm{g}$ per lane) were subjected to $10-12 \%$ SDS-PAGE and transferred to polyvinylidene fluoride membranes (EMD Millipore, Billerica, MA, USA). The membranes were then blocked with $5 \%$ non-fat milk for $2 \mathrm{~h}$, washed with PBS and incubated with following primary antibodies overnight at $4^{\circ} \mathrm{C}$ : Anti-PI3K antibody (cat. no. ab151549), anti-p-PI3K antibody (cat. no. ab182651; both 1:1,000 dilution; Abcam), anti-AKT antibody (cat. no. sc-377457) and anti-p-AKT antibody (cat. no. sc-33437; both 1:1,000 dilution; Santa Cruz Biotechnology, Inc., Dallas, TX, USA). An anti-GAPDH antibody (cat. no. ab9485; 1:2,000 dilution; Abcam) was used as a loading control. Subsequently, the membranes were incubated with a Goat Anti-Rabbit horseradish peroxidase conjugated secondary antibody (cat. no. ab6721; 1:2,000 dilution; Abcam) for $2 \mathrm{~h}$ at room temperature, and were visualized by an enhanced chemiluminescence system (Pierce; Thermo Fisher Scientific, Inc.). Western blot analysis was repeated at least three times.

Statistical analysis. Values are expressed as the mean \pm standard error of the mean. The data were analyzed by using Student's t-test or the one-way analysis of variance test. $\mathrm{P}<0.05$ was considered to indicate a statistically significant difference. Survival curves were analyzed by log-rank analysis (Kaplan-Meier method). GraphPad Prism (version 6.0; GraphPad Software Inc., La Jolla, CA, USA) was used for this analysis.

\section{Results}

Naringenin pre-treatment increases the survival rate of ALI mice. To assess the effect of naringenin on LPS-induced ALI, the survival rate of the mice was determined at $0,24,48,72$,

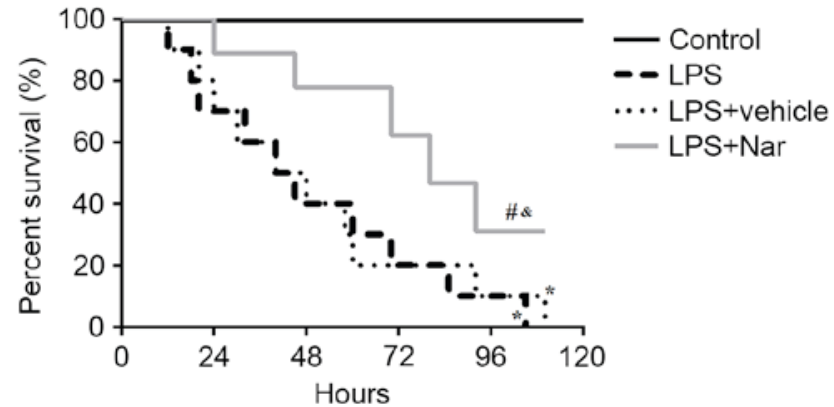

Figure 1. Effect of naringenin pre-treatment on survival rate. ${ }^{*} \mathrm{P}<0.05$ compared to the control group; ${ }^{\#} \mathrm{P}<0.05$ compared to the LPS-induced group; ${ }^{\&} \mathrm{P}<0.05$ compared to the vehicle-treated ALI group. Values are expressed as the mean \pm standard error of the mean. LPS, lipopolysaccharide; Nar, naringenin; ALI, acute lung injury.

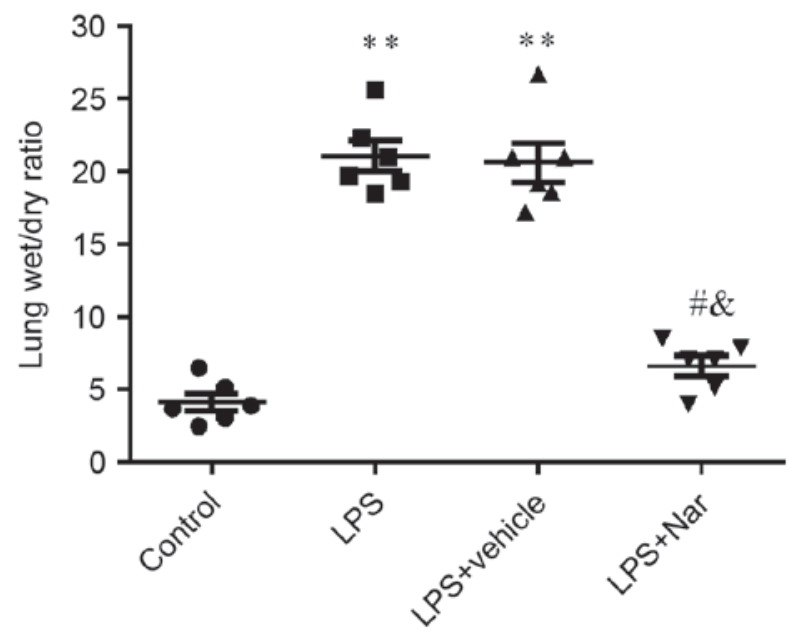

Figure 2. Effect of naringenin pre-treatment on lung wet/dry weight ratios. ${ }^{* *} \mathrm{P}<0.01$ compared to the control group; ${ }^{\text {}} \mathrm{P}<0.05$ compared to the LPS-induced group; ${ }^{\&} \mathrm{P}<0.05$ compared to the vehicle-treated ALI group. Values are expressed as the mean \pm standard error of the mean. LPS, lipopolysaccharide; Nar, naringenin; ALI, acute lung injury.

96 and $120 \mathrm{~h}$ after ALI. As shown in Fig. 1, the results showed that the survival rate was significantly improved by pre-treatment with naringenin compared with that in the LPS-induced or vehicle-treated ALI groups (both $\mathrm{P}<0.05$ ). The results indicated that pre-treatment with naringenin exerted a protective effect on LPS-induced ALI by improving the survival rate.

Naringenin pre-treatment inhibits ALI-induced increases in the lung wet/dry weight ratio. The mice were treated with LPS, with or without naringenin pre-treatment, and the lung wet/dry weight ratios were determined. The results showed that the lung wet/dry weight ratios were significantly higher in the LPS-induced or vehicle-treated ALI groups compared with that in the control group (both $\mathrm{P}<0.01$; Fig. 2). Pre-treatment with naringenin significantly decreased the lung wet/dry ratio compared with that in the LPS-induced or vehicle-treated ALI groups (both $\mathrm{P}<0.05$ ), indicating that naringenin decreased LPS-induced pulmonary edema.

Naringenin pre-treatment inhibits ALI-induced histological changes as well as increases in LDH activity, ALI score and 

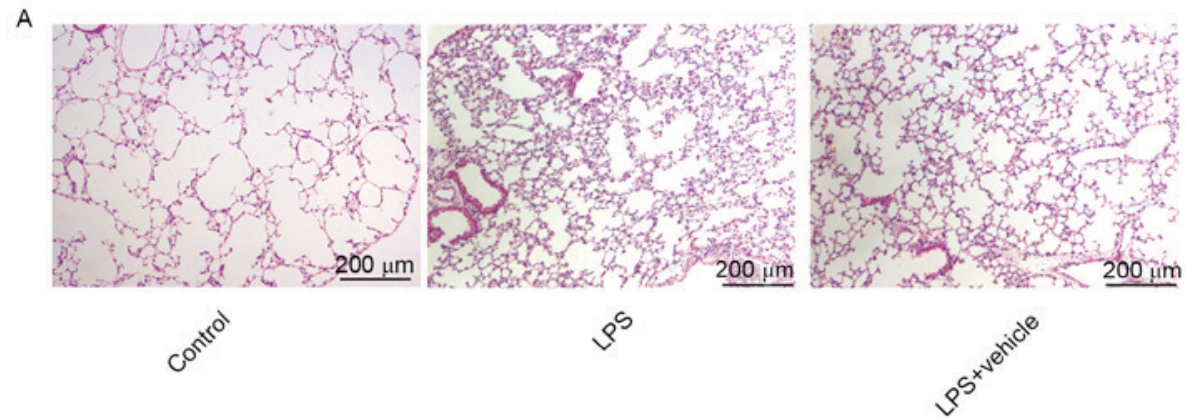

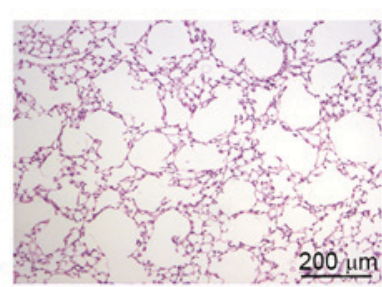

$8^{5^{x^{2}}}$
B

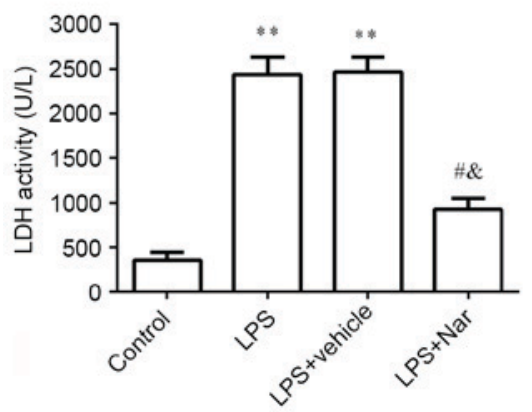

C

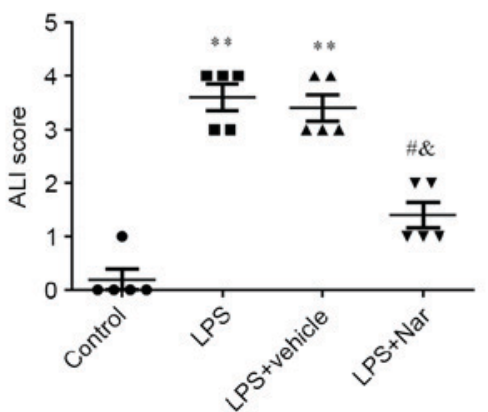

D

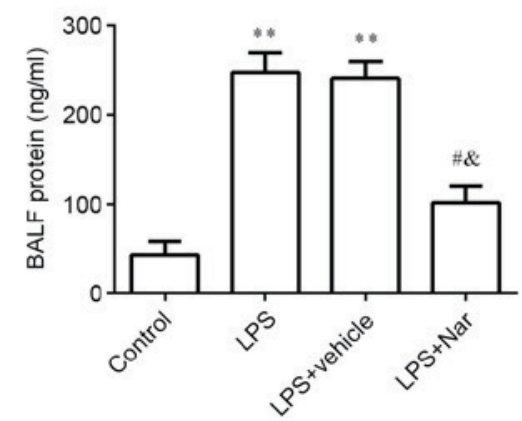

Figure 3. Effect of naringenin pre-treatment on (A) histological changes (scale bar, $200 \mu \mathrm{m}$ ), (B) LDH activity, (C) ALI score and (D) protein concentration in the BALF. ${ }^{* *} \mathrm{P}<0.01$ compared to the control group, ${ }^{, \mathrm{P}}<0.05$ compared to the LPS-induced group; ${ }^{\text {\& }} \mathrm{P}<0.05$ compared to the vehicle-treated ALI group. Values are expressed as the mean \pm standard error of the mean. LPS, lipopolysaccharide; Nar, naringenin; ALI, acute lung injury; BALF, bronchoalveolar lavage fluid; LDH, lactate dehydrogenase.
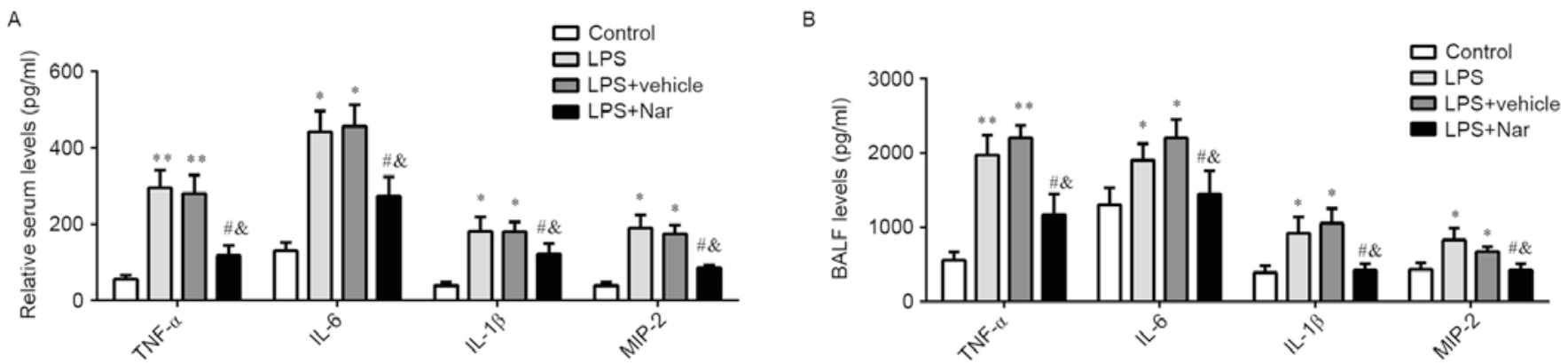

Figure 4. Effect of naringenin pre-treatment on inflammatory cytokines. (A) Serum levels and (B) BALF levels of TNF- $\alpha$, IL-6, IL-1 $\beta$ and MIP-2 in BALF. ${ }^{*} \mathrm{P}<0.05,{ }^{* *} \mathrm{P}<0.01$ compared to the control group, ${ }^{,} \mathrm{P}<0.05$ compared to the LPS-induced group; ${ }^{\circledR} \mathrm{P}<0.05$ compared to the vehicle-treated ALI group. Values are expressed as the mean \pm standard error of the mean. LPS, lipopolysaccharide; Nar, naringenin; ALI, acute lung injury; BALF, bronchoalveolar lavage fluid; TNF, tumor necrosis factor; IL, interleukin; MIP2, macrophage inflammatory protein 2.

protein concentration in the BALF. After treatment with naringenin and LPS induction, the morphology of the lung tissue was observed by histological examination. As indicated in Fig. 3A, several histopathological alterations were found in the LPS-induced and vehicle-treated ALI groups compared with the control group, including neutrophil infiltration, destruction of cellular structure, alveolar wall thickening and lung edema. Pre-treatment with naringenin markedly improved LPS-induced histopathological alterations, demonstrating that it exerted a protective effect against LPS-induced ALI. To further evaluate the protective role of naringenin in ALI, the LDH activity, ALI score and protein concentration in the lung BALF were evaluated. It was observed that LPS stimulation significantly increased LDH activity, ALI score and protein concentration in the lung BALF compared with the PBS-treated healthy control group (all $\mathrm{P}<0.01$ ). However, naringenin pre-treatment significantly decreased the above three indexes compared to those in the LPS-induced or vehicle-treated ALI groups (all $\mathrm{P}<0.05$; Fig. 3B-D). These results confirmed the preventive effect of naringenin against ALI and lung histological changes.

Naringenin pre-treatment reduces inflammatory cytokines in the serum and BALF after ALI. It has been well demonstrated that pro-inflammatory cytokines have a significant role in the pathogenesis of ALI (3). In the present study, the levels of TNF- $\alpha$, IL-6, IL-1 $\beta$ and MIP-2 were measured in the serum and BALF after treatment with naringenin and LPS induction. As shown in Fig. 4A and B, LPS treatment resulted in a significant increase in the serum and BALF levels of TNF- $\alpha$, IL-6, IL-1 $\beta$, and MIP-2 ( $\mathrm{P}<0.05$ or $\mathrm{P}<0.01)$. However, these LPS-stimulated elevations were inhibited by pre-treatment 

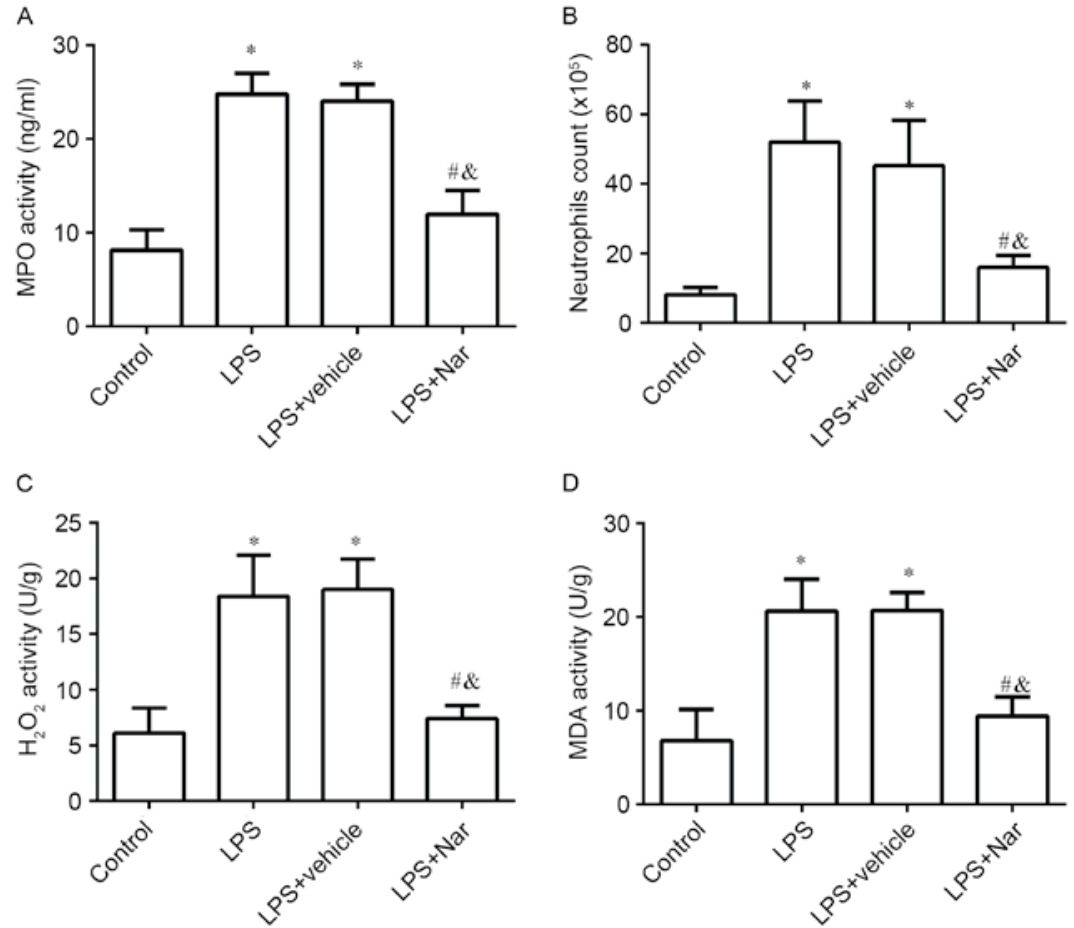

Figure 5. Effect of naringenin pre-treatment on MPO activity, neutrophil count and oxidative stress. (A) MPO activity; (B) neutrophil count; $(C) \mathrm{H}_{2} \mathrm{O}_{2}$, activity; (D) MDA activity in the different groups. ${ }^{*} \mathrm{P}<0.05$ compared to the control group; ${ }^{\#} \mathrm{P}<0.05$ compared to the LPS-induced group; ${ }^{\star} \mathrm{P}<0.05$ compared to the vehicle-treated ALI group. Values are expressed as the mean \pm standard error of the mean. LPS, lipopolysaccharide; Nar, naringenin; ALI, acute lung injury; MPO, myeloperoxidase; $\mathrm{H}_{2} \mathrm{O}_{2}$, hydrogen peroxide; MDA, malondialdehyde.

naringenin. Naringenin significantly decreased the levels of TNF- $\alpha$, IL-6, IL-1 $\beta$ and MIP-2 compared to those in the LPS-induced or vehicle-treated ALI groups (all $\mathrm{P}<0.05$ ). These results demonstrated that naringenin inhibited the inflammatory response in LPS-induced ALI.

Naringenin pre-treatment inhibits ALI-induced MPO activity, neutrophil count and oxidative stress. After treatment with naringenin, the MPO activity, neutrophil count and oxidative stress $\left(\mathrm{H}_{2} \mathrm{O}_{2}\right.$ and MDA activity) were determined. The results showed that LPS induction led to a significant increase in MPO activity (Fig. 5A), the number of neutrophils (Fig. 5B), $\mathrm{H}_{2} \mathrm{O}_{2}$ activity (Fig. 5C) and MDA activity (Fig. 5D) in the lung tissues, compared with those in the PBS-treated healthy control group (all $\mathrm{P}<0.05)$. However, pre-treatment with naringenin significantly decreased the MPO activity, the number of neutrophils, $\mathrm{H}_{2} \mathrm{O}_{2}$ activity and MDA activity compared with those in the LPS-induced ALI or vehicle-treated groups. In combination, these results indicated that naringenin attenuated the inflammatory and oxidative stress response in LPS-induced ALI.

Naringenin pre-treatment inhibits PI3K/AKT pathway activation in $A L I$. Previous studies have suggested that the PI3K/AKT pathway is involved in ALI $(11,22)$. To confirm whether naringenin exerts its protective effect on ALI is through the PI3K/AKT pathway, the total and the phosphorylated protein levels of PI3K and AKT in the lung tissues were determined. As shown in Fig. 6, the p-PI3K and p-AKT levels were significantly elevated by stimulation with LPS compared to those in the control group, which was significantly inhibited by pre-treatment with naringenin $(\mathrm{P}<0.05$; Fig. 6B). The results suggested that naringenin attenuated LPS-induced ALI through inhibition of the PI3K/AKT pathway.

\section{Discussion}

The present study found that naringenin pre-treatment significantly decreased the survival rate of mice with LPS-induced ALI, and inhibited histopathological changes, pulmonary edema and lung vascular leak. These effects are likely to be associated with the inhibition of ROS generation, neutrophil infiltration, and inflammatory cytokines levels in the serum and BALF by naringenin. In addition naringenin pre-treatment reduced the phosphorylated protein levels of PI3K and AKT. Therefore, it is concluded that naringenin pre-treatment ameliorates LPS-induced ALI through its anti-oxidative and anti-inflammatory activity and by inhibition of the PI3K/AKT pathway in mice.

It has been well demonstrated that ALI is strongly associated with infection with Gram-negative bacteria that release LPS (23). The manifestations of ALI in the LPS-induced mouse model are similar to the pathological characteristics of ALI in humans (24). Thus, LPS-induced ALI in mice appears to be a suited model for studying potential preventive or therapeutic strategies against ALI in humans. In the early phase of LPS-induced ALI, macrophages, neutrophils and other immune cells are stimulated by LPS and generate aberrant protein levels as well as release of inflammatory mediators in the BALF and serum, such as TNF- $\alpha$, IL-6, IL-1 $\beta$ and MIP-2 $(25,26)$. These inflammatory 

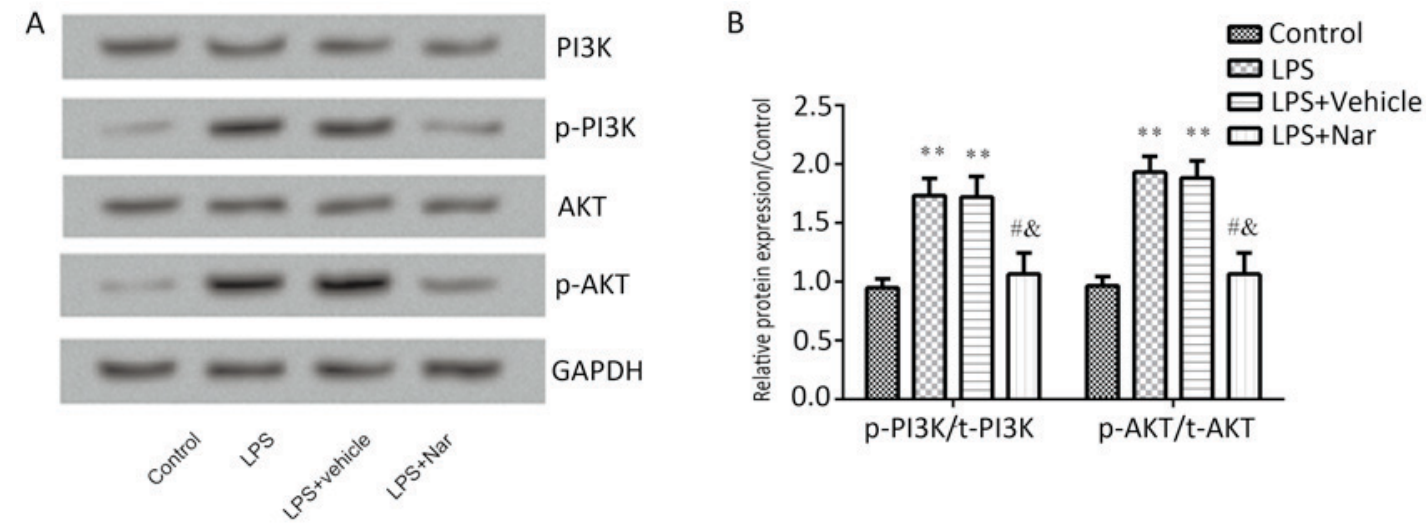

Figure 6. Effect of naringenin pre-treatment on the PI3K/AKT pathway. (A) Representative western blot images and (B) quantified expression levels of proteins. ${ }^{* *} \mathrm{P}<0.01$ vs. control group; ${ }^{\#} \mathrm{P}<0.05$ vs. LPS-induced group; ${ }^{\&} \mathrm{P}<0.05$ vs. vehicle-treated ALI group. Values are expressed as the mean \pm standard error of the mean. LPS, lipopolysaccharide; Nar, naringenin; p-PI3K, phosphorylated phosphatidylinostide-3-kinase.

mediators recruit polymorphonuclear neutrophils into the inflammatory lesions, largely contributing to the pathogenesis of ALI and ARDS $(27,28)$. MPO enzyme, an indicator of neutrophil accumulation in tissues, is mainly located in the primary granules of neutrophils. The activity of MPO indicates the adhesion and margination of neutrophils in the lung tissues (29); hence, MPO activation is considered to be a primary mechanism in the development of ALI (30). LDH activity in the BALF, an indicator of cellular damage and death, are increased and sustained following respiratory infection (31). Therefore, to determine the protective effect of naringenin against ALI, LPS-induced ALI mice were pre-treated with naringenin, and its effect on the survival rate, lung tissue wet/dry ratio, histological changes, LDH activity, ALI score, protein concentration in the BALF, MPO activity, and neutrophil count in the ALI mice was measured. The results of the present study showed that LPS-induced ALI reduced the survival rate, which was significantly increased by pre-treatment with naringenin, indicating a protective effect of naringenin on LPS-induced ALI. Edema is a typical symptom in response to systemic as well as local inflammation. The results regarding the lung tissue wet/dry ratio showed that pre-treatment with naringenin significantly inhibited ALI-associated increases in the ratio, demonstrating that naringenin relieved pulmonary edema. Moreover, the histopathological examination results showed that naringenin pre-treatment markedly improved the LPS-induced histological manifestations in LPS-induced ALI. Neutrophil infiltration, cell structure destruction and alveolar wall thickening in the naringenin group were reduced compared with those in the LPS-induced or vehicle-treated ALI groups. Furthermore, the LDH activity, ALI score and protein concentration in the lung BALF were all increased by LPS stimulation compared with those in the PBS-treated healthy control group, which was inhibited by naringenin pre-treatment. These results confirmed the protective effect of naringenin on lung histological changes.

Growing evidence supports that anti-inflammatory and anti-oxidant agents protect against LPS-induced ALI (32-34). LPS invokes the inflammatory response, subsequently resulting in increased production of ROS that are also responsible for LPS-induced ALI. In the present study, naringenin pre-treatment inhibited LPS-induced increases of TNF- $\alpha$, IL- $1 \beta$, IL- 6 , and MIP2, as well as MPO, neutrophil count, and $\mathrm{H}_{2} \mathrm{O}_{2}$ and MDA activity in BALF. These results were similar to those of Fouad et al (17), who suggested that naringenin pre-treatment against reduced LPS-induced ALI via its anti-inflammatory and anti-oxidant protective. In addition to the above results, the present study observed that the PI3K/AKT pathway was activated in ALI, which was consistent with the findings of previous studies that proved that the PI3K/AKT signaling pathway served as an important regulator of LPS-induced acute inflammatory responses in vitro and in vivo $(9,35)$. However, naringenin pre-treatment markedly decreased the ALI-induced phosphorylation of PI3K and AKT, indicating that naringenin pre-treatment protected against ALI by inhibition of the PI3K/AKT pathway.

In conclusion, the results of the present study suggested that naringenin pre-treatment ameliorates LPS-induced ALI in mice through its anti-oxidative and anti-inflammatory activity and by inhibition of the PI3K/AKT pathway.

\section{References}

1. Luh SP and Chiang $\mathrm{CH}$ : Acute lung injury/acute respiratory distress syndrome (ALI/ARDS): The mechanism, present strategies and future perspectives of therapies. J Zhejiang Univ Sci B 8: 60-69, 2007.

2. Rubenfeld GD, Caldwell E, Peabody E, Weaver J, Martin DP, Neff M, Stern EJ and Hudson LD: Incidence and outcomes of acute lung injury. N Engl J Med 353: 1685-1693, 2005.

3. Goodman RB, Pugin J, Lee JS and Matthay MA: Cytokine-mediated inflammation in acute lung injury. Cytokine Growth Factor Rev 14: 523-535, 2003.

4. Zhu GF, Guo HJ, Huang Y, Wu CT and Zhang XF: Eriodictyol, a plant flavonoid, attenuates LPS-induced acute lung injury through its antioxidative and anti-inflammatory activity. Exp Ther Med 10: 2259-2266, 2015.

5. Ware LB and Matthay MA: The acute respiratory distress syndrome. N Engl J Med 342: 1334-1349, 2000.

6. Ward PA: Oxidative stress: Acute and progressive lung injury. Ann N Y Acad Sci 1203: 53-59, 2010.

7. Imai Y, Kuba K, Neely GG, Yaghubian-Malhami R, Perkmann T, van Loo G, Ermolaeva M, Veldhuizen R, Leung YH, Wang $\mathrm{H}$, et al: Identification of oxidative stress and Toll-like receptor 4 signaling as a key pathway of acute lung injury. Cell 133: 235-249, 2008

8. Cachofeiro V, Goicochea M, de Vinuesa SG, Oubiña P, Lahera V and Luño J: Oxidative stress and inflammation, a link between chronic kidney disease and cardiovascular disease. Kidney Int Suppl: S4-S9, 2008. 
9. Guha M and Mackman N: The phosphatidylinositol 3-kinase-Akt pathway limits lipopolysaccharide activation of signaling pathways and expression of inflammatory mediators in human monocytic cells. J Biol Chem 277: 32124-32132, 2002.

10. Schabbauer G, Tencati M, Pedersen B, Pawlinski R and Mackman N: PI3K-Akt pathway suppresses coagulation and inflammation in endotoxemic mice. Arterioscler Thromb Vasc Biol 24: 1963-1969, 2004.

11. Lee JP, Li YC, Chen HY, Lin RH, Huang SS, Chen HL, Kuan PC, Liao MF, Chen CJ and Kuan YH: Protective effects of luteolin against lipopolysaccharide-induced acute lung injury involves inhibition of MEK/ERK and PI3K/Akt pathways in neutrophils. Acta Pharmacol Sin 31: 831-838, 2010.

12. Park HY, Kim GY and Choi YH: Naringenin attenuates the release of pro-inflammatory mediators from lipopolysaccharide-stimulated BV2 microglia by inactivating nuclear factor- $\kappa \mathrm{B}$ and inhibiting mitogen-activated protein kinases. Int J Mol Med 30: 204-210, 2012

13. Renugadevi J and Shagirtha K: In vivo and in vitro antioxidative efficacy of naringenin on cadmium-induced toxicity in rats. Res Rev A J Toxicol 3: 9-16, 2014.

14. Islas MS, Naso LG, Lezama L, Valcarcel M, Salado C, Roura-Ferrer M, Ferrer EG and Williams PA: Insights into the mechanisms underlying the antitumor activity of an oxidovanadium (IV) compound with the antioxidant naringenin. J Inorg Biochem 149: 12-24, 2015.

15. Li RF, Feng YQ, Chen JH, Ge LT, Xiao SY and Zuo XL: Naringenin suppresses K562 human leukemia cell proliferation and ameliorates Adriamycin-induced oxidative damage in polymorphonuclear leukocytes. Exp Ther Med 9: 697-706, 2015

16. Yu DH, Ma CH, Yue ZQ, Yao X and Mao CM: Protective effect of naringenin against lipopolysaccharide-induced injury in normal human bronchial epithelium via suppression of MAPK signaling. Inflammation 38: 195-204, 2015.

17. Fouad AA, Albuali WH and Jresat I: Protective Effect of Naringenin against Lipopolysaccharide-Induced Acute Lung Injury in Rats. Pharmacology 97: 224-232, 2016.

18. Starcher B and Williams I: A method for intratracheal instillation of endotoxin into the lungs of mice. Lab Anim 23: 234-240, 1989.

19. Numata M, Suzuki S, Miyazawa N, Miyashita A, Nagashima Y, Inoue $\mathrm{S}$, Kaneko $\mathrm{T}$ and Okubo $\mathrm{T}$ : Inhibition of inducible nitric oxide synthase prevents LPS-induced acute lung injury in dogs. J Immunol 160: 3031-3037, 1998.

20. Chen X, Yang X, Liu T, Guan M, Feng X, Dong W, Chu X, Liu J, Tian X, Ci X, et al: Kaempferol regulates MAPKs and NF- $\kappa$ B signaling pathways to attenuate LPS-induced acute lung injury in mice. Int Immunopharmacol 14: 209-216, 2012.
21. Simons RK, Maier RV and Chi EY: Pulmonary effects of continuous endotoxin infusion in the rat. Circ Shock 33: 233-243, 1991.

22. Kolliputi N and Waxman AB: IL-6 cytoprotection in hyperoxic acute lung injury occurs via PI3K/Akt-mediated Bax phosphorylation. Am J Physiol Lung Cell Mol Physiol 297: L6-L16, 2009.

23. Matthay MA, Ware LB and Zimmerman GA: The acute respiratory distress syndrome. J Clin Invest 122: 2731-2740, 2012.

24. Rojas M, Woods CR, Mora AL, Xu J and Brigham KL: Endotoxin-induced lung injury in mice: Structural, functional, and biochemical responses. Am J Physiol Lung Cell Mol Physiol 288: L333-L341, 2005.

25. Jerala R: Structural biology of the LPS recognition. Int J Med Microbiol 297: 353-363, 2007.

26. Lu YC, Yeh WC and Ohashi PS: LPS/TLR4 signal transduction pathway. Cytokine 42: 145-151, 2008.

27. Toews GB: Cytokines and the lung. Eur Respir J Suppl 34: 3s-17s, 2001.

28. Idell S: Anticoagulants for acute respiratory distress syndrome: can they work? Am J Respir Crit Care Med 164: 517-520, 2001

29. Jin SW, Zhang L, Lian QQ, Liu D, Wu P, Yao SL and Ye DY: Posttreatment with aspirin-triggered lipoxin A4 analog attenuates lipopolysaccharide-induced acute lung injury in mice: The role of heme oxygenase-1. Anesth Analg 104: 369-377, 2007.

30. Abraham E: Neutrophils and acute lung injury. Crit Care Med 31 (4 Suppl): S195-S199, 2003.

31. Ward PA, Till GO, Hatherill JR, Annesley TM and Kunkel RG: Systemic complement activation, lung injury, and products of lipid peroxidation. J Clin Invest 76: 517-527, 1985.

32. Wei D and Huang Z: Anti-inflammatory effects of triptolide in LPS-induced acute lung injury in mice. Inflammation 37: 1307-1316, 2014.

33. Sakaguchi M, Marutani E, Shin HS, Chen W, Hanaoka K, Xian M and Ichinose F: Sodium thiosulfate attenuates acute lung injury in mice. Anesthesiology 121: 1248-1257, 2014.

34. Zhang Z, Chen N, Liu JB, Wu JB, Zhang J, Zhang Y and Jiang X: Protective effect of resveratrol against acute lung injury induced by lipopolysaccharide via inhibiting the myd88-dependent Toll-like receptor 4 signaling pathway. Mol Med Rep 10: 101-106, 2014.

35. Xu CQ, Liu BJ, Wu JF, Xu YC, Duan XH, Cao YX and Dong JC: Icariin attenuates LPS-induced acute inflammatory responses: Involvement of PI3K/Akt and NF-kappaB signaling pathway. Eur J Pharmacol 642: 146-153, 2010. 